\title{
Fatores de risco e medidas de prevenção do câncer de próstata: subsídios para a enfermagem
}

\author{
Risk factors and prevention actions in prostate cancer: fundamentals for nursing
}

Factores de riesgo y medidas de prevención del cáncer de próstata: basis para la enfermería

\author{
Adriane Pinto de Medeiros", Maria de Fátima Batalha de Menezes", Anamaria Alves Napoleão' \\ 'Universidade Federal de São Carlos. Departamento de Enfermagem. São Carlos, SP \\ "Instituto Nacional do Câncer. Hospital do Câncer. Rio de Janeiro, RJ
}

Submissão: 25/02/2010

Aprovação: 27/07/2010

\section{RESUMO}

Apresenta-se um artigo de reflexão sobre fatores de risco e medidas de prevenção do câncer de próstata, visando contribuir para a abordagem de homens, especialmente durante a consulta de enfermagem. Idade avançada, origem étnica e hereditariedade são fatores de risco potenciais. Dieta pobre em gorduras, rica em frutas, verduras, legumes, grãos e outros é citada como minimizadora. A finasterida, droga inibidora da 5 alpha-redutase tem sido estudada como redutor de risco. O rastreamento da doença pela dosagem do Antígeno Prostático Específico (PSA) e toque retal é também medida recomendada. Entretanto, maiores evidências científicas ainda estão sendo buscadas sobre esses fatores. A abordagem dos homens na consulta de enfermagem pode contribuir para a identificação de fatores de risco, sinais e sintomas de possíveis alterações.

Descritores: Prevenção de doenças; Neoplasias; Neoplasias da próstata; Saúde do homem; Enfermagem.

\section{ABSTRACT}

A reflexive article about risk factors and prostate cancer prevention actions is presented to contribute to the approach of men, especially during nursing consultation. Increasing age, ethnical origin and heredity are potential risk factors. Low-fat diet and a diet rich in fruits, vegetables, legumes, grains and others, are related as capable of potentially minimize the risks. Finasteride, a drug that is inhibitor of 5 alpha-reductase has been studied as a risk reducer. Investigating the disease by measuring levels of Prostate-Specific Antigen (PSA) and digital rectal examination is also a recommended action. However, further scientific evidences about these factors are being sough. The approach of men in nursing consultation may contribute to the identification of risk factors, signs and symptoms of possible changes.

Key words: Disease prevention; Neoplasms; Prostatic neoplasms; Men's health; Nursing.

\section{RESUMEN}

Presentase un artículo de reflexión sobre los factores de riesgo y medidas de prevención del cáncer de la próstata, para contribuir en el tratamiento de los hombres, especialmente durante la consulta de enfermería. La edad avanzada, origen étnico y la herencia son factores de riesgo potenciales. Dieta baja en grasas y rica en frutas, verduras, legumbres, granos, entre otros está relacionada como potencialmente capaz de reducir los riesgos. Finasteride, un fármaco inhibidor de la 5 alfa-reductasa, se ha estudiado como un reductor de riesgo. El seguimiento de la enfermedad mediante la medición de los niveles de Antígeno Prostático Específico (PSA), y el examen digital del recto, son también recomendados. Sin embargo, si buscan más evidencias científicas acerca de estos factores. El enfoque de los hombres en la consulta de enfermería puede contribuir a la identificación de factores de riesgo, signos y síntomas de los posibles problemas.

Descriptores: Prevención de enfermedades; Neoplasias; Neoplasias de la próstata; Salud del hombre; Enfermería.

Resultados parciais de projeto de iniciação científica subvencionado pelo Programa Integrado de Bolsas de Iniciação Científica - Conselho Nacional de Desenvolvimento Científico e Tecnológico - Universidade Federal de São Carlos (PIBIC-CNPQ-UFSCar).

AUTOR CORRESPONDENTE Adriane Pinto de Medeiros. UFSCAR. Via Washington Luis, Km 235 - Monjolinho. CEP 13565-905. São Carlos, SP. E-mail: adriane_med@hotmail.com 


\section{INTRODUÇÃO}

De acordo com o Instituto Nacional de Câncer (INCA), órgão do Ministério da Saúde responsável pela prevenção e controle do câncer no Brasil, as estimativas para o ano de 2010 e válidas também para o ano de 2011 , apontam Que ocorrerão 489.270 casos novos de câncer. Os tipos mais incidentes, à exceção do câncer de pele do tipo não melanoma, serão os cânceres de pulmão e de próstata no sexo masculino ${ }^{(1)}$.

Em 2010, eram esperados 236.240 casos novos de câncer para o sexo masculino e estima-se Que os tumores mais incidentes para o sexo masculino serão devidos ao câncer de pele não melanoma (53 mil casos novos), seguido pelos tumores de próstata $(52 \mathrm{mil})^{(1)}$.

O câncer de próstata é o sexto tipo de câncer mais comum no mundo e o mais prevalente em homens, representando cerca de 10\% do total de câncer. Mais do Que Qualquer outro tipo de câncer, este é considerado o câncer da terceira idade, uma vez Que cerca de três Quartos dos casos no mundo ocorrem a partir dos 65 $\operatorname{anos}^{(2)}$.

No Brasil, o câncer de próstata é o mais incidente entre os homens, na Região Centro-Oeste (48/100.000). Exceto os tumores de pele não melanoma, esse tipo de câncer é o mais freQuente nas regiões Sul (69/100.000), Sudeste (62/100.000), Nordeste (44/100.000) e Norte $(24 / 100.000)^{(2)}$.

A mortalidade por câncer de próstata é relativamente baixa, o Que reflete, em parte, seu bom prognóstico Quando diagnosticado e tratado oportunamente. A sobrevida média mundial estimada em cinco anos é de 58\%. Nos países desenvolvidos, essa sobrevida passa para $76 \%$ e nos países em desenvolvimento $45 \%^{(2)}$.

Segundo a Sociedade Brasileira de Urologia (SBU), o câncer de próstata é o segundo maior causador de mortes no Brasil e estimase que 400 mil pessoas com mais de 45 anos tenham a doença e Que a maioria não tenha conhecimento disso. Anualmente, são diagnosticados 35 mil casos, com oito mil óbitos ${ }^{(3)}$.

No Que diz respeito à saúde dos homens em geral, é importante considerar Que, a cada três mortes de pessoas adultas, duas são de homens. Eles vivem, em média, sete anos menos do Que as mulheres e têm mais doenças do coração, câncer, diabetes, colesterol e pressão arterial mais elevadas ${ }^{(4)}$. Nesse sentido, é fundamental Que os profissionais da saúde estejam atentos aos problemas gerais e específicos Que podem acometer essa população, suas demandas de cuidados e acompanhem as políticas nacionais favorecedoras de sua atuação profissional.

Em relação às ações Que sinalizam para a preocupação relativa à saúde do homem no Brasil, conta-se com a Lei 10.289, de 20 de setembro de 2001 Que dispõe sobre a Instituição do Programa Nacional de Controle do Câncer de Próstata. Nela são estabelecidas, entre outras, as seguintes ações: I - campanha institucional nos meios de comunicação, com mensagens sobre o Que é o câncer de próstata e suas formas de prevenção; II - parcerias com as Secretarias Estaduais e Municipais de Saúde, colocando-se à disposição da população masculina, acima de Quarenta anos, exames para a prevenção ao câncer de próstata; III - parcerias com universidades, sociedades civis organizadas e sindicatos, organizando-se debates e palestras sobre a doença e as formas de combate e prevenção a ela; IV - outros atos de procedimentos lícitos e úteis para a consecução dos objetivos desta instituição - a Qual posterior- mente necessitou sofrer alterações no Parágrafo II do Art. $4^{\circ}$ para adequar-se a critérios técnico-científicos ${ }^{(5-6)}$.

Mais recentemente, a Política Nacional de Atenção Integral à Saúde do Homem, lançada em 2009 pelo Ministério da Saúde (MS), tem por objetivo facilitar e ampliar o acesso da população masculina aos serviços de saúde, em resposta à observação de Que os agravos do sexo masculino são um problema de saúde públi$\mathrm{Ca}^{(4)}$.

O Brasil será o primeiro país da América Latina e o segundo do continente americano a implementar uma política nacional de atenção integral à saúde do Homem (o primeiro foi o Canadá). A política encontra-se no contexto do Programa "Mais Saúde: Direito de Todos”, lançado em 2007 pelo Ministério da Saúde, Que visa promover um novo padrão de desenvolvimento focado no crescimento, bem-estar e melhoria das condições de vida do cidadão brasileiro $^{(4)}$.

A partir dos princípios e diretrizes da Política Nacional de Atenção Integral à Saúde do Homem, será possível orientar as ações e serviços de saúde para a população masculina, com integralidade e equidade, primando pela humanização da atenção, uma vez Que apresenta como princípios a humanização e a Qualidade, visando a promoção, reconhecimento e respeito à ética e aos direitos do homem, obedecendo às suas peculiaridades sócio-culturais ${ }^{(7)}$.

Diante dos dados em relação à morbimortalidade masculina $\mathrm{e}$ mais especificamente relativos ao câncer de próstata, entende-se Que essa iniciativa do MS brasileiro constitui um marco em relação à saúde do homem e certamente resultará em avanços significativos nesse sentido, com uma perspectiva de melhor Qualificação dos profissionais da saúde para atuarem nessa área e, conseQuentemente, no sentido de despertá-los para Questões voltadas especificamente à promoção da saúde do homem.

Diante do exposto, o propósito do presente artigo é apresentar uma reflexão sobre os fatores de risco e as medidas de prevenção de câncer de próstata, com vistas a contribuir com profissionais da saúde em geral e mais especificamente para oferecer subsídios aos enfermeiros na abordagem dos homens durante a consulta de enfermagem.

\section{FATORES DE RISCO E MEDIDAS DE PREVENÇÃO DO CÂNCER DE PRÓSTATA}

Para o levantamento das medidas de prevenção e fatores de risco existentes foram consultadas, no período de abril a junho de 2009, as bases de dados Scientifc Eletronic Library Online - SCIELO (www.scielo.br), Literatura da América Latina e Caribe - LILACS e Biblioteca Cochrane no sítio da Biblioteca Virtual em Saúde - BIREME (www.bireme.br). Também foram consultados os sítios do Instituto do Câncer INCA (www.inca.gov.br), Sociedade Brasileira de Urologia (SBU) (www.sbu.org.br), Society of Urologic Nurses and Associates (SUNA) (www.suna.org), European Association of Urology (EAU) (www.uroweb.org), além de documentos do Ministério da Saúde.

Em relação às medidas de prevenção do câncer de próstata, ainda não existem meios comprovadamente conhecidos para se prevenir este tipo de câncer ${ }^{(8-9)}$, da mesma forma como suas causas ainda permanecem desconhecidas ${ }^{(9-10)}$. Entretanto, ensaios clínicos estão sendo desenvolvidos com o objetivo de investigar melhor 
esse tópico ${ }^{(9)}$.

Fatores Que determinam o risco de desenvolvimento do câncer de próstata também não são bem conhecidos, apesar de alguns terem sido identificados ${ }^{(1)}$. Dentre eles destacam-se a idade avança$\mathrm{da}^{(5,10-13)}$, origem étnica - maior incidência em negros ${ }^{(9,10,13)}$ e origem hereditária ${ }^{(9-10,11-13)}$.

Neste contexto, considera-se mais especificamente que pai ou irmão com história de câncer de próstata ${ }^{(5,8,10)}$ antes dos 60 anos de idade ${ }^{(5,8,12)}$ pode aumentar o risco de desenvolver a doença de 3 a 10 vezes em relação à população em geral $^{(5,12)}$.

Marcadores exógenos são citados na literatura como agentes potencializadores ou minimizadores na determinação do risco do câncer de próstata. Dentre os Que possivelmente atuam na diminuição do risco destacam-se uma dieta rica em frutas,verduras, legumes ${ }^{(5,11,14)}$, vegetais ricos em carotenóides (por ex.: tomate e cenoura) $)^{(2,5,11,14)}$, grãos, cereais integrais e pobre em gordura, principalmente as de origem animal ${ }^{(5)}$.

Alguns componentes naturalmente encontrados nos alimentos possivelmente também possuem um efeito protetor como as vitaminas $A^{(5)}, D^{(2,5,11,14)}, E^{(2,5,11-14)}$, o selênio ${ }^{(2,5,11-14)}$ licopeno ${ }^{(2,11,13)}$, Ômega $3^{(2)}$, vitamina $C^{(11,13-14)}$. São ainda citados os fito-estrógenos, isoflavonóides, flavonóides, e lignanas ${ }^{(1)}$.

Já os fatores nutricionais citados como passíveis de influenciar potencialmente o desenvolvimento da doença incluem o total de energia consumida (refletida através do índice de massa corpo-

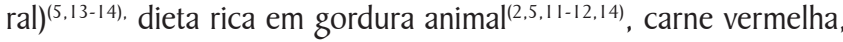
cálcio $^{(2,5,11,14,)}$, gorduras e leite ${ }^{(5)}$

Outros fatores como o tabagismo e o etilismo, vasectomia, além do fator de crescimento "insulin-like", também são citados como passíveis de influenciar potencialmente o desenvolvimento do câncer de próstata ${ }^{(5)}$. Algumas características específicas tais como a alta prevalência, longa latência, dependência endócrina, disponibilidade de marcadores de soro (PSA) e lesões precursoras histológicas (a neoplasia intra-epitelial prostática - PIN - é precursora do câncer de próstata invasivo) ${ }^{(11,14-16)}$, fazem do câncer de próstata um candidato ideal para medidas exógenas preventivas ${ }^{(11,14)}$.

Nesse sentido, mais estudos estão em andamento tentando esclarecer o papel dos fatores de risco e seu potencial para o sucesso na prevenção contra o câncer de próstata ${ }^{(14)}$. Sabe-se Que os fatores hereditários são importantes na determinação do risco de desenvolver clinicamente esse tipo de câncer, enquanto que os fatores exógenos podem ter um impacto importante nesse risco ${ }^{(1)}$.

Sobre a relação entre hábitos nutricionais e fatores de risco, evidências estão sendo acumuladas, mas autores referem Que ainda há um longo caminho a ser trilhado ${ }^{(15)}$.

Entre medidas farmacológicas de prevenção do câncer de próstata, consta na literatura estudo sobre o efeito da finasterida, droga inibidora da 5 alpha-redutase, em Que foi constatado seu potencial redutor no risco de câncer de próstata. Porém, sabe-se Que novos estudos buscando aperfeiçoamento desses resultados estão sendo conduzidos $^{(14,17)}$.

Outro dado importante é o de Que a incidência da doença assume diferentes valores de acordo com a região/área geográfica estudada, uma vez Que fatores exógenos afetam o risco da progressão da doença latente para sua forma clínica (p. ex. a doença possui alta incidência nos EUA e norte da Europa e baixa incidência no Sudoeste da Ásia) ${ }^{(11)}$.
No Que diz respeito à idade para início do rastreamento do câncer de próstata, observa-se peQuena variação em documentos consultados, uma vez Que tanto foi identificada a recomendação de Que seja realizado a partir dos 45 anos por homens Que não apresentam histórico familiar de câncer de próstata ${ }^{(3)}$, Quanto a de Que homens com idade igual ou maior Que 50 anos devem dar início à realização dos exames preventivos, sendo recomendado ainda Que, para homens com histórico familiar de câncer de próstata e afro-americanos, o rastreamento deve ter inicio aos 40 anos de idade $^{(9)}$.

Em relação à dosagem de PSA e toque retal, na literatura nacional, tanto foram identificadas recomendações de não se excluir a dosagem de PSA e o exame do toque digital como medidas de detecção precoce contra o câncer de próstata ${ }^{(3)}$, Quanto foram identificados documentos Que apontam Que não há evidências sobre o sucesso na redução da mortalidade com o rastreamento de pacientes pela dosagem de marcadores como o PSA e Que muitos homens podem estar sendo conduzidos a cirurgias desnecessárias ${ }^{(2)}$.

Apesar de algumas divergências terem sido identificadas e da afirmação de Que em geral sabe-se pouco sobre a maioria dos fatores estudados em relação ao câncer da próstata ${ }^{(7,9)}$, entende-se Que os profissionais devem buscar constantemente as melhores evidências disponibilizadas nos resultados de estudos e em documentos disponibilizados pelos órgãos oficiais que tratam dessas Questões; contribuir para a formulação de protocolos com base nesses estudos ou documentos e seguir condutas com base nas melhores evidências e em discussões e decisões interdisciplinares em seus locais de trabalho.

\section{CONSIDERAÇÕES FINAIS}

Há elementos Que possibilitam aos enfermeiros e demais profissionais da saúde uma abordagem em relação aos fatores de risco e fatores exógenos relativos ao câncer de próstata junto aos homens.

Mais especificamente na área da enfermagem, a recente legislação Que dispõe sobre a Sistematização da Assistência de Enfermagem (SAE) e a implementação do Processo de Enfermagem estabelece como função privativa do enfermeiro a Consulta de Enfermagem e resolve Que o Processo de Enfermagem deve ser realizado, de modo deliberado e sistemático, em todos os ambientes, públicos ou privados, em Que ocorre o cuidado profissional de Enfermagem $^{(18)}$.

Dessa maneira, o enfermeiro não deve perder a oportunidade de abordar os homens, aproveitando as situações cotidianas da assistência de enfermagem, na perspectiva da promoção da saúde e detecção precoce de agravos, no sentido de orientá-los sobre os fatores de risco e medidas de prevenção relativas ao câncer de próstata, além de identificar a presença ou não desses fatores e buscar sinais e sintomas Que possam indicar alterações relacionadas.

Com essa iniciativa, espaços de fomento à promoção da saúde do homem podem ser promovidos, inclusive nas situações do cuidado hospitalar, o que é pertinente na medida em Que os homens, diferentemente das mulheres, procuram os serviços, na maioria das vezes, para atendimentos de emergência e não de forma deliberada e programada para manutenção da saúde ${ }^{(19)}$.

Diante do exposto, sugere-se que os profissionais, nos difer- 
entes espaços de cuidado, acompanhem os resultados de estudos Que apresentem melhores níveis de evidência em termos de prevenção do câncer de próstata e fatores de risco, o Que também poderá oferecer subsídios para a elaboração e atualização de protocolos e condutas a serem seguidos.

Considera-se necessário também Que novas pesQuisas sejam conduzidas na área da promoção da saúde e prevenção do câncer, bem como Que ocorram mudanças na educação formal da população e no ensino específico dos profissionais da área da saúde, uma vez Que estes podem proporcionar uma maior adesão aos programas de promoção da saúde e prevenção do câncer pelos indivíduos ${ }^{(20)}$.

\section{REFERÊNCIAS}

1. Ministério da Saúde (BR). Instituto Nacional de Câncer. Estimativa 20 I0: Incidência de Câncer no Brasil: Introdução. Rio de Janeiro: INCA; 2010.

2. Ministério da Saúde (BR). Instituto Nacional de Câncer. Estimativa 20 10: Incidência de Câncer no Brasil: Síntese de Resultados e Comentários. Rio de Janeiro: INCA; 2010.

3. Sociedade Brasileira de Urologia. Campanha Dia Nacional de Combate ao Câncer da Próstata. [on line]. 2005. [citado em em 2009 Mar 09]. Disponível em: http:/www.sbu.org. br/ indexGeral.php?do $=$ imprensa\&sub $=7 \&$ dado $\_$id $=86 \&$ site $=$ geral

4. Ministério da Saúde (BR). Notícias. MS lança Política Nacional de Saúde do Homem. [on line]. 2009. [citado em 2009 Nov 27]. Disponível em: http://portal.saude.gov.br/portal/aplicacoes/noticias/default.cfm?pg =dspDetalheNoticia\&id_a rea $=124 \&$ CO_NOTICIA $=10490$

5. Ministério da Saúde (BR). Instituto Nacional de Câncer. Programa nacional de controle do câncer da próstata: documento de consenso. Rio de Janeiro (RJ): INCA, 2002. [citado em 2009 Abr 17]. Disponível em: http://bvsms.saude.gov.br/bvs/ publicacoes/cancer_da_prostata.pdf

6. Brasil. Lei n. 10.289, de 20 de setembro de 2001. Dispõe sobre a Instituição do Programa Nacional de Controle do Câncer de Próstata.Legislação Federal. 2001. [citado em 2010 Fev 08]. Disponível em: http://www.planalto.gov.br/ccivil 03/Leis/ LEIS_200I/LI0289.htm

7. Ministério da Saúde (BR). Secretaria de Atenção à Saúde. Departamento de Ações Programáticas Estratégicas. Política Nacional de Atenção Integral à Saúde do Homem: Princípios e Diretrizes. Brasília: Ministério da Saúde; 2008.

8. Ministério da Saúde (BR). Instituto Nacional de Câncer. Prevenção do câncer de próstata: normas e recomendações do INCA. Rev Bras Cancerol 2003; 49(4): 204.

9. Society of Urologic Nurses and Associates. Prostate Cancer: Patient fact sheet.[on line]. [cited on 2009 Nov 16]. Available from: http://www.suna.org/members/prostate_cancer.pdf

10. Torti DC, Matheson GO. Exercise and prostate cancer. Sports Med 2004; 34(6): 363-69.
1 1. European Association of Urology. Guidelines on Prostate Cancer. [on line]. [cited on 2009 Nov 16]. Available from: http:// www.uroweb.org/fileadmin/tx_eauguidelines/2009/Full/ Prostate_Cancer.pdf

12. Ministério da Saúde (BR). Instituto Nacional de Câncer. Prevenção e controle do câncer: normas e recomendações do INCA. Rev Bras Cancerol 2002; 48(3): 317-32.

13. Friedenreich CM, Thune L. A review of physical activity and prostate câncer risk. Cancer Cases Control 200 I; 12: 46 I-75.

14. Schmid HP, Engeler DS, Pummer K, Schmitz-Dräger B J. Prevention of prostate cancer: more Questions than data. Cancer Prevention. Recent Results Cancer Res 2007; 174: I0I-7.

15. Schulman CC, Zlotta AR, Denis L, Schroder FH, Sakr WA. Prevention of prostate cancer. Scand I Urol Nephrol 2000; 205(sup): 50-61.

16. Presti JC. Neoplasias da próstata. In: Tanagho EA, McAninch IW. Urologia geral de Smith. 16 ${ }^{\mathrm{a}}$. ed. Barueri: Manole; 2007. p. $406-28$.

17. Timothy W. 5-alpha-reductase inhibitors for prostate cancer prevention. Cochrane Database of Systematic Reviews. In: The Cochrane Library, Issue 2, Art. No. CD00709 I. [cited on 2009 Jul 7]. http://cochrane.bvsalud.org/cochrane/main.php? lang $=$ pt\&lib $=$ COC

18. Conselho Federal de Enfermagem (Brasil). Resolução Cofen358/2009. [online]. Dispõe sobre a Sistematização da Assistência de Enfermagem e a implementação do Processo de Enfermagem em ambientes, públicos ou privados, em Que ocorre o cuidado profissional de Enfermagem, e dá outras providências. Brasília-DF, 15 de outubro de 2009. [citado em 2010 Fev 3]. Disponível em: http://www.portalcofen.gov.br/ 2007/materias.asp?ArticleID $=10113 \&$ sectionID $=34$

19. Paiva EP. Conhecimentos, atitudes e práticas acerca da detecção do câncer de próstata. Rio de laneiro: Universidade Federal do Rio de laneiro/Escola de Enfemagem Anna Nery; 2008.

20. Cestari MEW, Zago MMF. A prevenção do câncer e a promoção da saúde: um desafio para o Século XXI. Rev Bras Enferm 2005; 58(2): 218-21. 